\title{
Effectiveness of Knowledge Management Functions in Improving the Quality of Education in Higher Education Institutions
}

\author{
Shafqat Hameed and Atta Badii
}

\begin{abstract}
Knowledge management provides connections between areas such as information technology, leadership, organisational behaviour and strategy which are very important in education sector. The aim of this study is to identify how effective knowledge management is in higher education institutions of Pakistan. A multi method approach is used to collect data for this study. This study presents the main factors and principles desired and required for successful implementation of knowledge management in higher education institutions.
\end{abstract}

Index Terms-Knowledge, management, technology, education, pakistan

\section{INTRODUCTION}

Knowledge can be described as the information which is linked to convey certain meaning. Management of these insubstantial resources and potentials helps the organization to generate competitiveness among other rivals in the market. The organization capability to recognize vital knowledge resources and utilize them objectively in solving the problem boosts competence, promotes self-confidence and directs to achieve productivity. "KM is concerned with making the right knowledge available to the right processor such as human or computer, at the right time in the right presentations for the right cost". (Holsapple, Joshi, 1999). It is difficult to distinguish between the concepts of knowledge management (KM) and organizational learning (OL) owing to their common characteristics (Bijaya Mishra and A. Uday Bhaskar, 2011). Duncan and Weiss (1978) defined learning as a "process in which knowledge forms both input and output". Other researchers like Kogut and Zander (1992), Nonaka and Takeuchi (1995) and Spender (1996) shared the same opinion. KM then, is a "set of techniques and practices which eases the flow of knowledge into and within the firm" Birkinshaw (2001). Formation of knowledge in an organization is dependent upon the perception of the organization as to the way it learns (Scho“n, 1975). As per Bijaya Mishra and A. Uday Bhaskar (2011), an organization can be termed as LO if it displays performance in KM practices / processes. Based on existing reviews of $\mathrm{KM}$ processes, they established four main categorizations / a theme of KM processes i.e. knowledge

Manuscript received May 23, 2012; revised June 17, 2012.

S. Hameed is with National University of Sciences and Technology (NUST), Islamabad, Pakistan (e-mail: Shafqat.hameed@ceme.nust.edu.pk).

A. Badii is with University of Reading, UK (e-mail: atta.badii@reading.ac.uk). creation, knowledge up-gradation, and knowledge sharing and knowledge retention. Going a step further, they made a comparison between two organizations basing on these themes. The purpose was to find out the degree of learning in these organizations. This study also uses these themes (in addition to other factors described below) for evaluating the learning process in selected higher education institutions of Pakistan.

American Productivity and Quality Center and Arthur Anderson Business Consulting (1999) designed a knowledge management appraisal tool (KMAT) in 1995 in order to assess the 'operational performance' of KM. KMAT is helpful for various organizations in choosing specific type of KM suitable to their working. KMAT is comprised of following basic components:

- Technology

- Leadership and strategy

- KM process

- Culture

- Measurement

Besides, following key success factors were suggested:

- Information technology used in KM

- Adopted procedures of KM

- $\mathrm{KM}$ organizational structure in support

- Human resource committed in KM

However, as stated by Y.-F. Wen (2009), in the present scenario, it may be hard to determine a wide-ranging and precise reference criteria for $\mathrm{KM}$ owing to the fact that standardized KM methods and structure are yet to be defined.

A categorization of organization wide $\mathrm{KM}$ activities as carried out by Allee (1997) is given below:

1) Knowledge creation

2) Knowledge retention

3) Knowledge sharing

4) Knowledge innovation

However, Allee (1997) considered only the organizational KM activities and did not take into account their "effectiveness". As per Hoy and Miskel (2001), following are the phases into which the effectiveness measurement of organizational activity can be organized:

- Knowledge capability effectiveness

- Knowledge adaptation effectiveness

- Knowledge integration effectiveness

- Knowledge accomplishment effectiveness

Certain measures for effectiveness of KM were also suggested by Fernandez and Sabherwal (2001) and Wen (2001). An operational effectiveness of KM was given by Sveiby (2002). Storck and Hill (2000), Levett and Guenov (2000), and Gold et al. (2001) have put forward some evaluation tables. According to Housel and Bell, the most 
significant manifestation is what we receive in the feedback.

The presence of $\mathrm{KM}$ factors in any organization is correlated to effectiveness of KM practices which is further correlated to improvement in the learning process. The same is depicted below in Fig. 1.

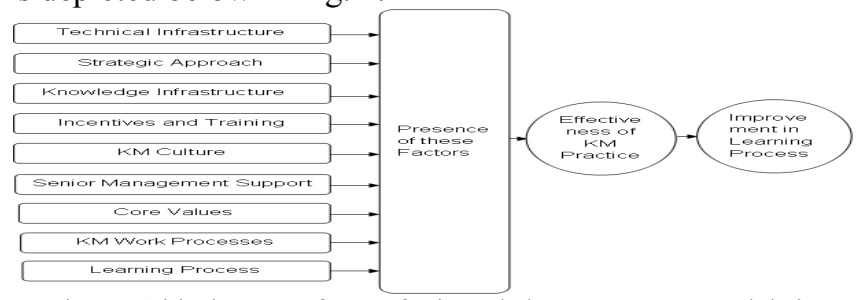

Fig. 1. Critical success factors for knowledge management and their relationship with effectiveness of KM practices and improvement in learning process

The presence of these critical success factors ultimately leads to the effectiveness of $\mathrm{KM}$ practices in higher education institutions which ultimately leads to the improvement in learning process. In order to achieve desired results, these factors are critical success factors (CSF) and, therefore, have to be taken into account.

In the absence of appropriate Information Technology (IT) tools i.e. networks, portals, databases /repositories, computers, software, KMS experts, there cannot be any implementation of $\mathrm{KM}$ as IT is the most essential ingredient for implementation of KM. Development of a proper strategy for implementing KM . (Skryme \& Amidon, 1997), (Liebowitz, 1999), (Wong, 2005). Knowledge formation and collaboration should be properly knitted all across an organization. There must be a proper alignment between the organization's KM framework and operational processes while incorporating all performance criteria. (Davenport et al, 1998). Rewards are considered vital in order to promote a behavior of knowledge creation and sharing. Many authors proposed a system of rewards as CSF like incentives to encourage knowledge sharing (Liebowitz, 1999). Besides rewards, imparting of proper training and education to employees on KM related activities is believed to be of quite significance. This training is equally essential for both low level employees as well as top management (Wong, 2005). In any organization, importance of a knowledge friendly culture is of paramount importance. In fact, a knowledge friendly culture lies at the heart of KM implantation. (Davenport et al, 1998; Skryme \& Amidon, 1997).

\section{RESEARCH METHODOLOGY}

A multi method approach was adopted combining closed and open ended questions in the questionnaire. Main issues of consideration in the questionnaire included views on following:

1) Existence of various knowledge management (KM) themes / processes e.g. knowledge acquisition and creation, knowledge upgradation, knowledge sharing and distribution etc in the HEI

2) Extent of application of various KM factors e.g. knowledge strategy, technical infrastructure, KM infrastructure, incentives and training, knowledge supporting culture, senior management support etc in the HEI.

3) Effectiveness of $\mathrm{KM}$ practices in improving the learning process in the HEI.

4) Impediments to effective implementation of KM in the HEI and measures to improve upon the learning process etc.

5) Best ways of bringing $\mathrm{KM}$ in the organizational culture.

A sample size of 400 was chosen. Accordingly, a total of 400 questionnaires were distributed in person to the five universities between December 2011 and February 2012. 291 persons responded, giving a response rate of 72 percent. This response rate is found satisfactory as it is in line with Meile and Dobratz (1995) who set themselves a response rate target of $50-60 \%$.

Within each issues of consideration, a five point Lickerttype scale was used for closed ended questions. The aim was to determine effectiveness of $\mathrm{KM}$ practices in improving the learning process in higher education institutions of Pakistan. The scale asked the respondent to tick a mark 1 if they strongly disagreed to a particular question up to a mark of 5 if they strongly agreed to a given question. The open ended part of the questionnaire asked the respondents to give biggest three hurdles in effective implementation of KM in the HEI, best three ways to bring $\mathrm{KM}$ in the organisational culture and best three factors for effective improvement of the learning process.

In order to subject the data to statistical testing, the collected data were coded and analyzed using SPSS (originally Statistical Package for the Social Sciences) version 17 for Windows). The data were also analyzed using percentages calculated through MS Excel.

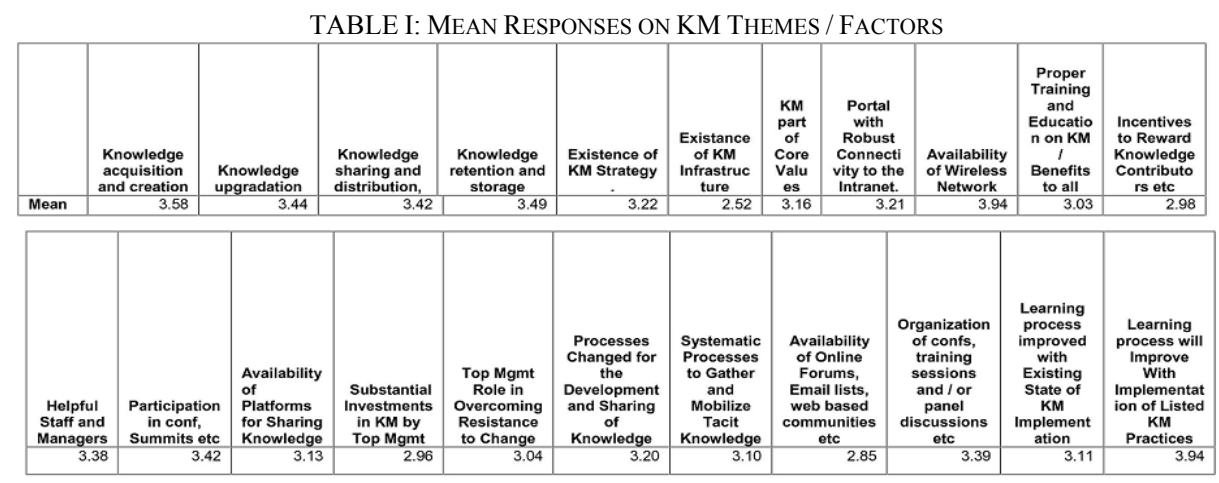




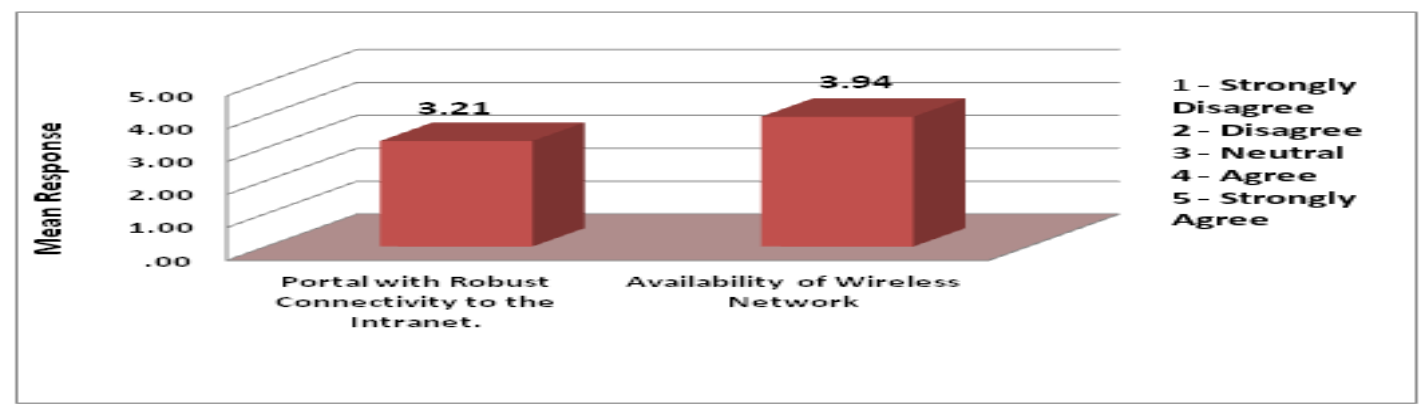

Fig. 2. Mean response

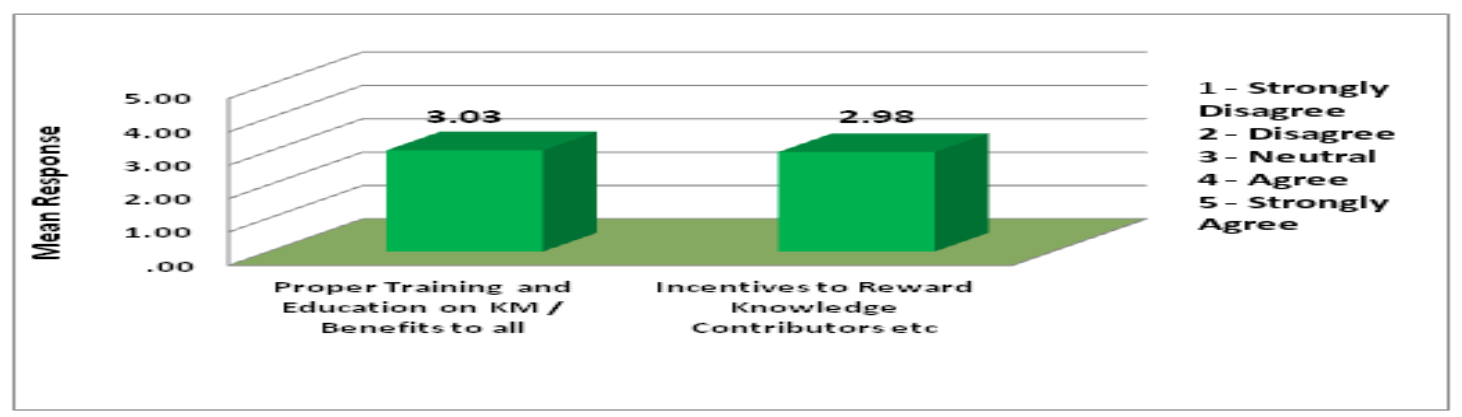

Fig. 3. Mean response

The results illustrate existence of KM themes in HEIs as viewed by the respondents. It is evident that mean response on all KM themes fluctuates arround 3.5 which implies limited existence of these themes in HEIs. However, people were not as certain on the presence of a portal with robust connectivity to the intranet. The extent of training and education imparted on KM practices / benefits in HEIs is considered an impoirtant factor as well as the presence of any incentives in HEIs to reward knowledge contributors, top innovative thinkers etc. Results explains the existance of KM supporting culture in HEIs as suggested by the respondents through their input on related activities. A general disagreement was found on the availability of proper platforms / systems in HEIs to share knowledge. Other factors such as participation in conferences, seminars etc and willingness of staff / mangers to extend help on request was found to exist to some extent only. Results describe respondents view on top management support in KM development in HEIs. This important support was considered scanty both in terms of the investment initiatives on $\mathrm{KM}$ as well as playing a role in overcoming resistance to change due to KM implementation. As per the respondents, in HEIs, the work processes were changed to a very limited extent to encourage development and sharing of knowledge. Besides, the implementation of systematic processes to gather and mobilize tacit knowledge was considered a grey area. While there was a partial agreement on the limited holding of conferences, seminars, educational summits, a relative disagreement was found on the availability of online forums, email lists, web based communities etc in HEIs.

People agreed in unequivocal terms on the non availability of a KM infrastrucure in HEIs whereas only limited presence of a KM strategy was consented. Similarly, the survey results showed that KM is emphasized in the vision and mission statements of the HEIs to a limited extent only. Majority of the respondents thought that the learning process in HEIs has improved partially with the existing state of $\mathrm{KM}$ implementation. More so, the vast majority of the respondents showed their conviction that learning process will improve if KM implementation takes place in totality.

From the survey data, it is quite evident that knowledge management themes i.e. knowledge creation, upgradation, sharing and retention exist in HEIs to some extent only. Birkinshaw (2001) stated that most organizations today are building networks that support knowledge acquisition and distribution through continuous learning. Hence, successful firms (organizations) are those that are better at learning, or in other words, they are those that are better at sharing knowledge among individuals It can therefore be inferred from the above statement that effectiveness of learning process in an organization (in this study, an HEI) is directly linked with the existence / application of above defined KM themes.

Although knowledge management is in application in the modern world for quite some time now, it is relatively a new concept in Pakistan. Hence, one of the most important factors in the effective KM implementation in Pakistan HEIs is the basic understanding of what is knowledge management, why it is required and what are the associated benefits. Unless the top management in the higher education sector and HEIs has an answer to these basic questions and is fully convinced to apply the concept of $\mathrm{KM}$ in the HEI processes, the desired level of effectiveness of the KM practices and resultant improvement in the leaning process cannot be achieved. This argument was amply complemented by majority of the respondents in the survey who considered lack of understanding of $\mathrm{KM}$ and its benefits to be one of the biggest hurdles in effective implementation of knowledge management.

The survey results have clearly shown the level of application of KM themes / factors in the HEIs as perceived by the respondents as discussed above in detail. The same is summarized below:

1) All KM themes have a limited application only. 
2) With regards to technical infrastructure, wireless networks are mostly available. However, portals with connectivity to the intranet, knowledge repositories and document management systems are inadequately available.

3) KM culture is applied to some degree only.

4) Existence of KM Strategy is upto a certain level which is well below the required degree. Similarly, application of KM as a core value in the vision and mission statements of HEIs is also well below the required level.

5) Availability of a KM infrastructure, Incentive and training on KM, senior management support to KM application, KM work processes and organization of learning activities have been indicated as extremely weak areas and require immediate attention.

Above mentioned summary clearly indicates a very limited and unsatisfactory application of KM themes and factors in the HEIs. In order to proceed further, opinion of the respondents was sought as to whether the learning process in HEIs has improved with the existing state of KM implementation. Mean response to this question was 3.11 which fell short of a clear agreement. Thus, with an unsatisfactory state of KM application in the HEIs as viewed by the respondents, there is an unsatisfactory mean response on improvement in learning process. This clearly supports our argument i.e. learning process is linked to the extent to which the knowledge management practices are applied in HEIs. The underlying argument was further strengthened by the respondents when they overwhelmingly agreed that a befitting implementation of all KM factors will definitely improve learning process in the HEIs.

\section{CONCLUSION AND RECOMMENDATIONS}

Based upon the survey findings on KM themes / factors application in HEIs, importance of various KM factors and impediments to $\mathrm{KM}$ implementations, following is recommended in the order of priority in order to improve upon the effectiveness of KM practices in HEIs for corresponding increase in the learning process:

1) All KM themes / processes exist in HEIs to a limited extent only. These themes are required to be incorporated in HEIs more effectively to achieve enhanced learning. In order to attain this objective at the micro level, a focused approach to employ following KM factors in the given order is solicited:

2) Top management support and commitment is the most important factor for KM implementation in HEIs which is extremely lacking. Concerted efforts should be made to provide this critical support mainly in terms of financial investment initiatives, an active role in improving upon $\mathrm{KM}$ understanding and overcoming resistance to change due to KM implementation.

3) There is an urgent need to improve technical infrastructure in HEIs for effective KM implementation. This requires mainly the availability of portals with connectivity to the intranet, knowledge repositories and document management systems etc. Besides, availability of wireless network in HEIs is adequate which needs to be maintained.

4) Incentives and training on KM practices are almost non existent in HEIs. There is a dire need to impart proper training and education on KM practices / benefits to all levels of employees including top management with special emphasis on IT training. Besides, commercial or non commercial incentives are required to be introduced in HEIs to reward knowledge contributors, top performers, top innovative thinkers etc.

5) Most HEIs do not have a proper KM strategy in place. HEIs in Pakistan need to formulate and implement proper KM strategy which should identify knowledge users, sources, processes and storage strategy.

6) The existence of work processes for knowledge capture and use was found well below the required level. This warrants due implementation of systematic processes in HEIs to gather and mobilize tacit knowledge. Moreover, existing work processes in HEIs are required to be changed in order to incorporate development and sharing of knowledge.

7) The survey results clearly indicate non existence of $\mathrm{KM}$ infrastructure in HEIs. For effective KM implementation, such infrastructures need to be established in HEIs in the form of a KM department and Chief Knowledge / Information Officer.

8) An organizational culture supporting learning, sharing and use of knowledge is an important element for KM implementation. KM supporting culture exists in HEIs to a limited extent only. In order to improve upon this aspect, staff / managers are required to be more willing to give advice or help on request. Moreover, there is a need to generate a conducive environment in HEIs for participation in conferences, seminars, educational summits, training sessions, panel discussions. Existence of proper platforms or systems to share knowledge was relatively found the weakest area and need immediate actions.

9) Non availability of online forums, email lists; web based communities and / or chat rooms to encourage virtual communities of practice have been found an extremely weak area. These learning activities are required to be established and organized in HEIs in order to increase effectiveness of the KM practices and improve the learning process

\section{REFERENCES}

[1] B. Mishra and U. Bhaskar, "Emerald Group Publishing Limited," Journal of Knowledge Management, vol. 15, no. 2, pp. 344-359, ISSN 1367-3270, 2011.

[2] C. W. Holsapple and K. D. Joshi, "Knowledge selection: Concepts, issues, and technologies," Handbook on Knowledge Management, Shaw M et al. (eds). Springer-Verlag: New York, 1999.

[3] B. Kogut and U. Zander, "Knowledge of the firm, combinative capabilities, and the replication of technology", Organisation Science, vol. 3, no. 3, pp. 383-97, 1992.

[4] I. Nonaka, and H. Takeuchi, "The Knowledge-Creating Company: How Japanese Companies Create the Dynamics of Innovation," Oxford University Press, New York, NY, 1995. 
[5] J. Birkinshaw, "Making sense of knowledge management," Ivey Business Journal, vol. 65, no. 4, pp. 32-6, 2001.

[6] D. Scho" n, "Deutero-learning in organisations: learning for increased effectiveness," Organisation Dynamics, vol. 4, no. 1, summer, pp.2-16, 1975.

[7] Y. F. Wen, "An effectiveness measurement model for knowledge management," Knowledge- Based Systems, vol. 22, pp. 363-367, 2009.

[8] Y. F. Wen, "A study on the key success factors of knowledge management," Report of 2001 Special Research Project, National Science Council, Taiwan, 2001.
[9] American Productivity and Quality Center (APQC), Knowledge Management Consortium Benchmarking Study: Final Report, American Productivity and Quality Center, Houston, Texas.

[10] A. Allee, "12 Principles of knowledge management, Training and Development," vol. 51, no. 11, pp. 71-74, 2000.

[11] G. P. Levett and M. D. Uenov, "A methodology for knowledge management implementation," Journal of Knowledge Management, vol. 4, no. 3, pp.258-270, 2000. 